\title{
Prevalencia al nacimiento de microtia-anotia. Maternidad del Hospital Clínico de la Universidad de Chile, período 1983-2005
}

\author{
Julio Nazer $\mathbf{H}^{1}$, G uillermo Lay-Son $\mathbf{R}^{2 a}$, Lucía Cifuentes $\mathbf{0}^{3}$. \\ Prevalence of microtia and anotia at \\ the Maternity of the University of \\ Chile Clinical Hospital
}

Background: Microtia is a congenital defect characterized by disturbances in the size and form of the ear lobe. Anotia corresponds to the absence of the ear lobe. Aim: To study the prevalence of microtia and anotia at the Maternity of the University of Chile Clinical Hospital. Material and methods: Analysis of the database of the Latin American Collaborative Study of Congenital Defects (ECLAMC). All newborns and stillborns with congenital defects are incorporated to this database. Results: The prevalence of microtia-anotia in the period 1982-2001 was 8.7 per 10,000 born alive. Chilean hospitals have an uniform prevalence of 5.2 per 10,000 born alive. Thirty seven percent presented as isolated malformations and the rest were associated to other defects. Eighty six percent of non isolated cases were part of a syndrome. Sixty eight percent were mild or moderate forms and the rest, severe forms. Two cases were stillborns and two newborns died before hospital discharge. Conclusions: The prevalence of microtia in this hospital and in the rest of Chilean hospitals is significantly higher than in the rest of non Chilean hospitals participating in the ECLAMC, that is 4.1 per 10,000 born alive (Rev Méd Chile 2006; 134: 1295-301).

(Key words: Anotia; Hereditary motor and sensory neuropathies; Microtia; Nervous system malformations)

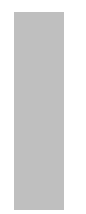

$\mathrm{E}^{1}$ término microtia se refiere a una anomalía congénita del oído externo, ya sea en su tamaño o en su morfología, que puede presentarse en forma variable desde un pabellón auricular más pequeño, hasta la ausencia completa del pabellón (anotia). Este espectro de anomalías

Correspondencia a: Dr. Julio Nazer H. Unidad de Neonatología, Departamento de Obstetricia y Ginecología, Facultad de Medicina, Hospital Clínico Universidad de Chile. Santiago de Chile. E mail: jnazer@redclinicauchile.cl puede asociarse a atresia del conducto auditivo externo y del oído medio.

Tiene una gran connotación social, ya que las personas afectadas pueden sufrir de problemas psicológicos derivados de este defecto, visible y difícil de ocultar y, además, alteraciones funcionales relacionadas donde un alto porcentaje de ellas sufren de un déficit importante de la audición, lo que obliga a tratamientos especializados y multidisciplinarios.

Embriológicamente, el oído externo, junto al oído medio, nervio facial, maxilar superior e inferior, 
se origina a partir del primer y segundo arco branquial, lo que explica la asociación de la microtia con las anomalías del espectro EOAV (espectro óculo-aurículo-vertebral), el síndrome de Treacher Collins y la secuencia de Pierre Robin. El pabellón auricular se desarrolla a partir de tres prominencias mesenquimatosas denominadas montículos, prominencias auriculares, que surgen de las márgenes del primer arco branquial. El mesénquima de estas prominencias proviene del mesodermo del primer y segundo arcos branquiales. El núcleo o centro del mesoderma, que forma el área primitiva del conducto auditivo, puede alterarse al producirse la recanalización y provocar una atresia de él. Al mismo tiempo que se desarrolla anormalmente el primer arco branquial, otras estructuras derivadas de él, como el tensor del tímpano, maléolo, el incus y la mandíbula, pueden también alterarse. Al comprometerse el segundo arco branquial, puede producirse algunas anomalías del nervio facial ${ }^{1,2}$.

Comúnmente, la microtia se expresa en grados de I a IV, en el cual el grado IV corresponde a la anotia. Es una anomalía de diagnóstico obvio, por lo que las diferencias de frecuencias entre los distintos centros seguramente son reales y no debido a falta de diagnóstico o a sobrerregistros. La microtia puede presentarse en forma aislada 0 como parte de un síndrome malformativo.

Un gran porcentaje de esta patología se presenta en forma aislada, esporádica dentro del grupo familiar, compatibles con una herencia multifactorial. Se han descrito familias en las que la herencia ha sido autosómica recesiva o autosómica dominante ${ }^{3}$. Las formas asociadas, a menudo tienen una causa genética, formando parte de síndromes, asociaciones o secuencias malformativas, entre los cuales destacan el espectro óculoaurículo-vertebral (que engloba a la microsomía hemifacial y el síndrome de Goldenhar), el síndrome de Treacher-Collins y cromosomopatías, como las trisomías 13 y 18.

La etiología de esta anomalía es desconocida, pero se sugiere que el mecanismo de producción sea una disrupción, una alteración del flujo vascular durante el desarrollo ${ }^{4,5}$, pero lo más común son casos esporádicos compatibles con una herencia multifactorial ${ }^{6}$. Entre los factores ambientales, la microtia se ha asociado con el uso de ciertos teratógenos como la talidomida ${ }^{7,8}$ el ácido retinoico ${ }^{9}$, el alcohol y la diabetes mellitus ${ }^{10,11}$.

\section{OBjetivos}

Debido a que existen escasos estudios que caracterizan la epidemiología de la microtia, el presente trabajo tiene como objetivo establecer la prevalencia al nacimiento de las distintas formas de microtia en la maternidad del Hospital Clínico de la Universidad de Chile en el período entre enero de 1983 y julio de 2005 y compararlas con las cifras de los otros hospitales chilenos participantes del ECLAMC, con el resto del ECLAMC (ECLAMC sin Chile) y con otros programas de monitorización de malformaciones congénitas a nivel mundial, calcular la frecuencia de casos aislados y asociados a otras malformaciones, síndromes reconocidos, precisar la frecuencia de atresia del conducto auditivo externo, si son uni o bilaterales, y estudiar diversos factores demográficos, como peso de nacimiento, sexo, edad materna, otros malformados en la familia, y consanguinidad.

\section{PACIENTES Y MÉTODO}

El ECLAMC (Estudio Colaborativo Latino Americano de Malformaciones Congénitas) ${ }^{12}$ es un sistema de registro de investigación y de vigilancia epidemiológica, de base hospitalaria y de tipo caso-control, para estudiar la prevalencia al nacimiento y los factores de riesgo para anomalías congénitas, que se inició en 1967. Chile, por intermedio del Hospital Clínico de la Universidad de Chile (HCUCH), ingresó en 1969 y desde esa fecha ha permanecido en él, en forma activa e ininterrumpida. Para comparar nuestros resultados con los otros hospitales chilenos del ECLAMC y con el total del ECLAMC, recurrimos al último Informe Final del ECLAMC, publicado en 2002 y con material de 1982 a $2001^{13}$.

Todos los recién nacidos (RN) son examinados por un pediatra neonatólogo de la maternidad. A este registro se incorpora todo recién nacido $(\mathrm{RN})$ vivo (NV) o mortinato (NM) que nace en la maternidad del Hospital, que pese 500 gramos 0 más y que presente una 0 más malformaciones congénitas. Se completa una ficha ad hoc, según un Manual Operacional ${ }^{14}$, en la que se consignan los antecedentes maternos, controles prenatales, datos sobre el parto y del RN, como peso al nacimiento, sexo, nacido vivo o muerto y se describe detallada- 
mente las malformaciones que presenta. Se toma como control al RNV, del mismo sexo, que nace a continuación y que no presenta anomalías congénitas. Los NM malformados no tienen control. Así se ha formado una base de datos, de la que extrajimos la muestra para este trabajo.

Basado en las características del defecto, clasificamos cuatro grupos, de acuerdo al Manual Operacional del ECLAMC ${ }^{14}$ :

- Microtia I. La oreja es malformada y más pequeña que lo normal. Es posible reconocer todas las partes de la oreja.

- Microtia II. La oreja es más pequeña y menos desarrollada que en el grado I; el hélix no está totalmente desarrollado. Las otras partes de la oreja pueden no estar bien definidas.

- Microtia III. Sólo queda un cordón, el borde del hélix y pueden quedar segmentos sueltos, no identificables.

- Microtia IV. Anotia: ausencia total de la oreja.

De acuerdo a si era posible identificar el conducto auditivo externo, se les clasificó como acompañados o no de atresia.

Estudiamos el período 1 de enero de $1983 \mathrm{al}$ 31 de julio de 2005.

Se compararon las tasas de prevalencia al nacimiento mediante prueba de chi cuadrado.

\section{RESULTADOs}

En el período 1 de enero de 1983 a 31 de julio de 2005, se produjeron 65.053 nacimientos consecutivos en la Maternidad del HCUCH, 64.841 RNV y 462
NM. Esto representa una mortinatalidad global de 0,71\%. Entre ellos se encontraron $4.528 \mathrm{RN}$ malformados (6,96\% del total de nacimientos), $4.462 \mathrm{NV}$ (6,88\%) y $66 \mathrm{NM}(14,28 \%$; 66/462). En el total de RN malformados, 57 (1,25\%) presentaron algún grado de anotia-microtia, lo que representa una prevalencia al nacimiento de 8,76 por 10.000 (Tabla 1). Estas frecuencias son mayores que las encontradas por otros autores ${ }^{15}$. Al comparar la prevalencia al nacimiento de microtia entre los distintos hospitales chilenos, se aprecian significativas diferencias $\left(\mathrm{X}^{2}\right.$ $=46,03 ; \mathrm{p}=0,0044)$ que se deben a que el Hospital del Salvador y San José de Santiago, Hospital Dr. E. Torres de Iquique y Gustavo Fricke de Viña del Mar presentan tasas significativamente más elevadas que el resto de hospitales chilenos. La prevalencia de microtia en Chile (5,2 por 10.000) es significativamente superior al resto del ECLAMC $(4,1$ por 10.000 ; $\mathrm{X}^{2}=10,74 ; \mathrm{p}=0,001$ ) (Tabla 2).

En la Tabla 3 se compara la prevalencia al nacimiento de microtia en distintos países del mundo y se observa que existen grandes diferencias entre los distintos centros ( $X^{2}=577,7$ con 15 grados de libertad; $p<0,001$ ) consistentes en mayores prevalencias en poblaciones latinoamericanas y mucho menores en poblaciones caucásicas y japonesa, lo cual es concordante con lo señalado por otros autores ${ }^{16-18}$.

En 21 pacientes (36,8\%) el defecto se presentó en forma aislada y en 36 (63,2\%) estaba asociado a otras anomalías congénitas, esto representa una tasa de 3,23 por mil para las formas aisladas y 5,53 por mil para las asociadas.

En 21 casos formaban parte de algún síndrome conocido y 10 casos en que no se especificó el

Tabla 1. Total de nacimientos, nacidos vivos y mortinatos, total de pacientes con anotiamicrotia, en NV y M N . M aternidad del H ospital C línico U niversidad de C hile. Período 1983-2005

\begin{tabular}{|lrrc|}
\hline \multicolumn{1}{c}{$\mathrm{n}$} & \multicolumn{1}{c|}{$\%$} & Tasa x 10.000 \\
\hline Total de nacimientos & 65.053 & & \\
Nacidos vivos & 64.841 & & \\
Nacidos muertos & 462 & 0,71 & \\
Total de malformados & 4.528 & 6,96 & \\
Malformados vivos & 4.462 & 6,88 & \\
Malformados mortinatos & 66 & 14,93 & \\
Total de RN con microtia & 57 & -- & 8,76 \\
RNV con microtia & 55 & -- & 8,48 \\
RNM con microtia & 2 & -- & 4,33 \\
\hline
\end{tabular}


diagnóstico del síndrome, es decir 86,1\% (31/36) de los casos asociados formaban parte de algún síndrome, los más frecuentes fueron trisomía 18 y síndrome de Goldenhar (Tabla 4). Esta proporción difiere en parte de otras comunicaciones, en que algunos dan mayor frecuencia a las aisladas ${ }^{17}$. En 5 pacientes $(8,8 \%)$ se asoció únicamente a papilomas preauriculares y fístulas auriculares.

Cuando analizamos individualmente las anomalías que con mayor frecuencia se asociaron a microtia en nuestra muestra, tanto en formas sindrómicas como no sindrómicas, destacan además de los papilomas preauriculares y fístulas auris, las alteraciones de la conformación de los huesos craneanos y las anomalías del desarrollo mandibular. Varios de los niños afectados de microtia, presentaban más de una malformación asociada (Tabla 5).

En 40 pacientes $(70,2 \%)$ la anomalía fue unilateral, siendo más frecuente a derecha (65\%).

En cuanto a la severidad del defecto, $39 \mathrm{RN}$ $(68,4 \%)$ presentaron formas leves a moderadas (21 pertenecían al grupo I de la clasificación y 18 al grupo II). Mientras que 18 niños (31,6\%) presentaban formas severas, 15 eran del grupo III y 3 pacientes presentaron anotia (grupo IV). Veinticinco $\mathrm{RN}(43,9 \%)$ presentaban atresia del orificio de entrada al conducto auditivo externo, lo que constituye un signo de severidad de la anomalía.

Tabla 2. Total de nacimientos, de casos con microtia y tasas por 10.000 en hospitales chilenos participantes en el ECLAM C y en el total del ECLAM C Período 1982-2001

\begin{tabular}{|llccc|}
\hline Hospital & Ciudad & Nacimientos & №de Microtia & Tasa x 10 mil \\
\hline Clínico Universidad de Chile Santiago & 63.656 & 34 & 5,3 \\
Clínica C. Freire & Santiago & 3.942 & 1 & 2,5 \\
Dr. Juan Noé & Arica & 3.419 & 0 & 0,0 \\
Regional & Valdivia & 46.437 & 23 & 5,0 \\
Higueras & Talcahuano & 76.566 & 31 & 4,0 \\
Clínica Las Condes & Santiago & 14.846 & 7 & 4,7 \\
Militar & Santiago & 9.845 & 5 & 5,1 \\
Grant Benavente & Concepción & 53.344 & 26 & 4,9 \\
Naval & Valparaíso & 13.246 & 4 & 3,0 \\
Clínica Francesa & Concepción & 6.417 & 3 & 4,7 \\
Gustavo Fricke & Viña del Mar & 10.623 & 11 & 10,4 \\
Clínica S. Alemán & Valdivia & 9.257 & 7 & 7,6 \\
Clínica Tabancura & Santiago & 1.581 & 2 & 12,7 \\
Del Profesor & Santiago & 1.169 & 0 & 0,0 \\
Regional & Rancagua & 22.399 & 12 & 5,4 \\
Dr. E. Torres & Iquique & 2.046 & 4 & 19,6 \\
Hospital Base & Linares & 5.753 & 0 & 0,0 \\
Hospital de Puerto Montt & Puerto Montt & 5.944 & 4 & 6,7 \\
Sótero del Río & Santiago & 15.331 & 5 & 3,3 \\
Salvador & Santiago & 12.129 & 15 & 12,4 \\
San Borja Arriarán & Santiago & 13.474 & 4 & 3,0 \\
San José & Santiago & 15.260 & 14 & 9,2 \\
Regional & Talca & 4.087 & 1 & 2,4 \\
Hospital de Cauquenes & Cauquenes & 349 & 0 & 0,0 \\
Hospital de Curicó & Curicó & 1.048 & 1 & 9,5 \\
Total de Chile & & 412.168 & 1.290 & 5,2 \\
Resto del ECLAMC & & 1.504 & 4,1 \\
Total del ECLAMC & 3.162 .441 & & 4,2 \\
\hline
\end{tabular}


Tabla 3. Comparación de anotia-microtia entre Programas de Registro de M F (ICBD M S Anual Report 1992)

\begin{tabular}{|lr|}
\hline País & Tasa x 10.000 \\
\hline Ecuador: Quito & 17,40 \\
Chile: Santiago & 8,76 \\
México & 6,22 \\
Chile & 5,10 \\
Eclamc & 4,96 \\
Finlandia & 3,69 \\
Suecia & 2,50 \\
España & 2,05 \\
Israel & 1,89 \\
Italia & 1,46 \\
China & 1,40 \\
USA: Atlanta & 1,28 \\
Australia & 1,09 \\
Japón & 0,90 \\
Dinamarca & 0,70 \\
Francia: París & 0,50 \\
Inglaterra y Gales & 0,25 \\
Rep. Checa & 0,25 \\
Noruega & 0,17 \\
\hline
\end{tabular}

\section{Tabla 4. Síndromes reconocidos en los que se encontró microtia}

\begin{tabular}{|lrr|}
\hline Síndrome & \multicolumn{2}{c|}{$\mathrm{N}^{\circ}$ de casos } \\
\hline Trisomía 18 & $4 / 57$ & $(7 \%)$ \\
Goldenhar & $4 / 57$ & $(7 \%)$ \\
Treacher-Collins & $3 / 57$ & $(5,3 \%)$ \\
Microsomía hemifacial & $3 / 57$ & $(5,3 \%)$ \\
Trisomía 13 & $1 / 57$ & $(1,8 \%)$ \\
Orofaciodigital & $1 / 57$ & $(1,8 \%)$ \\
Secuencia de Pierre Robin & $1 / 57$ & $(1,8 \%)$ \\
Holoprosencefalia alobar & $1 / 57$ & $(1,8 \%)$ \\
Siameses polimalformados & $1 / 57$ & $(1,8 \%)$ \\
Branquio-oto-renal? & $1 / 57$ & $(1,8 \%)$ \\
CHARGE & $1 / 57$ & $(1,8 \%)$ \\
Subtotal & 21 & $(36,8 \%)$ \\
Asociación papiloma-fístula & $5 / 57$ & $(8,8 \%)$ \\
N/E & $10 / 57$ & $(17,5 \%)$ \\
Total & $36 / 57$ & $(50,9 \%)$ \\
\hline
\end{tabular}

Referente a la distribución por sexo, no hubo diferencias (29 femeninos y 28 masculinos). Sin embargo, la proporción de sexos es diferente en las formas aisladas que en las asociadas, aunque esta diferencia no alcanza la significación estadística ( $p=0,37)$ : en las mujeres, $31 \%$ de los casos la microtia estaba asociada a otras anomalías, en tanto que esto ocurrió en $42,8 \%$ de los varones.

El peso promedio fue de 2.928 gramos, inferior al peso promedio de los controles 3.289 gramos, lo que está de acuerdo a lo encontrado por otros autores $^{17}$. El promedio de la edad materna de los $\mathrm{RN}$ afectados fue de 28,1 años y el de los controles 28,5 años.

$\mathrm{Al}$ investigar por otros malformados en la familia, este antecedente se encontró en 27 casos (47,4\%).

En cinco casos (8,8\%) encontramos antecedentes de otros miembros de la familia afectados con anomalías del EOAV.

Al revisar la mortalidad entre los niños con microtia, encontramos que 2 de los 57 recién nacidos con microtia (3,5\%) fueron mortinatos. Y que de los restantes 55 nacidos vivos, fallecieron 8 posteriormente $(14,5 \%)$.

De los mortinatos, 1 niño tenía un síndrome polimalformativo no reconocido y 1 presentaba trisomía 18. De los recién nacidos con microtia que fallecieron en el servicio, 4 eran polimalformados, 1 era portador de una trisomía 13, 1 con trisomía 18 y 1 con holoprosencefalia alobar.

\section{DisCUSIÓN}

Un hecho importante que conviene destacar cuando se analizan frecuencias de alguna malformación congénita, es la variedad de criterios diagnósticos que pueden haber entre los distintos registros. Esto mismo ocurre con las prevalencias de anotia-microtia. El ECLAMC, cuya metodología seguimos nosotros, por lo que son comparables, entrega cifras que van desde 1\%o en Passo Fundo, Brasil hasta 19,05 por mil y 20,8 por mil en dos maternidades de La Paz, Bolivia ${ }^{13}$, lo que demuestra que en un mismo registro las frecuencias son heterogéneas entre los hospitales.

Se puede observar, también, esta misma heterogeneidad entre los distintos hospitales chilenos participantes en el ECLAMC (Tabla 2), discrepancias sobre las que no tenemos una explicación, con excepción 


\section{La Tabla 5. Anomalías más frecuentemente asociadas con microtia en nuestro material}

\begin{tabular}{|lcc|}
\hline Anomalías asociadas & Frecuencia & Porcentaje \\
\hline Papiloma preauricular & $11 / 57$ & $19,3 \%$ \\
Alteraciones huesos craneales & $11 / 57$ & $19,3 \%$ \\
Micrognatia-agnatia & $10 / 57$ & $17,6 \%$ \\
Fisura paladar y/o labio & $8 / 57$ & $14,0 \%$ \\
Cardiopatía congénita & $8 / 57$ & $14,0 \%$ \\
Alteración extremidades inferiores & $8 / 57$ & $14,0 \%$ \\
Alteración extremidades superiores & $8 / 57$ & $14,0 \%$ \\
Genitales hipoplásicos & $7 / 57$ & $12,3 \%$ \\
Alteraciones en tamaño boca & $7 / 57$ & $12,3 \%$ \\
Alteraciones en nariz & $6 / 57$ & $10,5 \%$ \\
Alteraciones palpebrales & $6 / 57$ & $10,5 \%$ \\
Alteraciones renales & $5 / 57$ & $8,8 \%$ \\
Asimetría facial & $5 / 57$ & $8,8 \%$ \\
Fístula auris & $4 / 57$ & $7,0 \%$ \\
Alteraciones oculares & $4 / 57$ & $7,0 \%$ \\
Alteraciones neuroanatómicas & $4 / 57$ & $7,0 \%$ \\
\hline
\end{tabular}

tal vez de los hospitales que tienen una muestra muy pequeña, lo que explicańa sus muy altas o muy bajas tasas, debido sólo al azar. Anotia-microtia es una anomalía externa, de fácil diagnóstico, por lo que es poco probable que hubiera un subregistro o sobremegistro en una patología de diagnóstico obvio.

Si comparamos nuestras frecuencias con las de otros programas a nivel mundial (Tabla 4), se puede observar que las tasas más altas son de poblaciones hispánicas o indoamericanas (Chile, ECLAMC, México, España), con la única excepción de Finlandia, por lo que International Clearinghouse for Birth Defects Monitoring Systems ${ }^{15}$, sugiere que existe una importante contribución genética en la etiología de esta anomalía congénita. En un estudio de Aase y cols ${ }^{16}$, en la población de Nuevo México, encontraron que la microtia es significativamante más frecuente en la población indoamericana que en los otros grupos raciales estudiados. Un aporte a esta hipótesis es lo publicado por Castilla ${ }^{17}$, quien propone que, además de la influencia genética, existe otro factor importante que es la altura sobre el nivel del mar, ya que las áreas de mayor prevalencia de esta anomalía congénita están por sobre los 1.500 metros de altura sobre el nivel del mar. Quito (2.819 metros) es la ciudad con la más alta tasa descrita de microtia $(17,4$ por 10.000$)$, a ello hay que agregar Ciudad de México (2.309 metros) con una tasa de 6,22 por 10.000 , las tasas también son relativamente altas en Bogotá, Colombia (2.700 metros) y La Paz, Bolivia (3.640 metros).

Un hecho interesante encontrado en nuestra muestra, es la menor frecuencia de casos aislados, comparados con lo encontrado por MartínezFrías $^{18}$ del Estudio Colaborativo Español de Malformaciones Congénitas (ECEMC) que usó también la metodología ECLAMC, que reportó $55,7 \%$ de formas aisladas, contra 36,8\% de la nuestra. Otto Sánchez y cols ${ }^{19}$ refieren $47,3 \%$ de formas aisladas en Ciudad Bolívar, Venezuela, Castilla y Orioli17 encontraron en Quito, Ecuador, $85 \%$ de casos aislados y sólo $15 \%$ asociados y en el resto de los países del estudio, 66,3\% de casos aislados y 33,7\% de asociados. De los casos aislados, $87,5 \%$ tenían atresia del orificio de entrada al conducto auditivo externo, de los asociados sólo el $52,8 \%$. Shaw et $\mathrm{al}^{20}$ encontraron una tasa de 2,16 por mil de anotia-microtia en niños sin anomalías cromosómicas ( 0,63 por mil aisladas y 1,53 mil asociadas a otras anomalías estructurales). Encontraron también mayor prevalencia aisladas entre hispánicos y asiáticos que entre blancos no hispánicos. Mastroiacovo et $\mathrm{als}^{6}$ encontraron mayor prevalencia entre mujeres primíparas, en tanto que Harris et als $^{21}$ describen 
mayor prevalencia entre mujeres grandes multíparas, en varones e hispánicos. Otros factores de riesgo comunicados son, además de la altura sobre el nivel del mar, el tabaquismo paterno y la diabetes insulino dependiente ${ }^{9-11}$.

Es interesante presentar las características genealógicas de algunos casos en los que encontramos parientes afectados con anomalías del espectro óculo-aunículo-vertebral (EOAV), compatibles con herencia mendeliana autosómica dominante.

En un caso de microtia tipo II derecha sin atresia de CAE, de sexo masculino, su padre presentaba una microtia de grado similar, además de un papiloma preauricular.

En otro caso de microtia tipo I derecha, de sexo masculino, el abuelo paterno tuvo malformación de orejas no especificadas, una tía paterna con anotia

\section{REFERENCIAS}

1. KeITH L Moore. Embriología Clínica 4⿳a Edición. 1989. Ed Interamericana pp 457-

2. Malformaciones de las orejas. En Malformaciones congénitas. Ed ME Hübner, R Ramírez, J. Nazer. Editorial Universitaria. Santiago. Chile. 2005 p. 86.

3. Sánchez-Corona J, García-Cruz D, Ruenes R, Cantu JM. A distinct dominant form of microtia and conductive hearing loss. Birth Defects Orig Artic Ser 1982; 18: 211-6.

4. ORSTAVIK KH, MEDBo S, MaIR IW. Right-sided microtia and conductive hearing loss with variable expressivity in three generations. Clin Genet 1990; 38: 117-20.

5. Poswiшo DE. The pathogenesis of first and second branchial arch syndrome. Oral Surgery 1973; 35: 302-28.

6. Mastroiacovo P, Corchia C, Botto LD, Lanni R, Zampino G, Fusco D. Epidemiology and genetics of microtia-anotia: a registry based study on over one million births. J Med Genet 1995; 32: 453-7.

7. TaKemori S, Tanaka Y, SuzUKi JI. Thalidomide anomalies of the ear. Arch Otolaryngol 1976; 102: 425-7.

8. SmTtheLs RW, NeWMAN CG. Recognition of thalidomide defects. J Med Genet 1992; 29: 716-23.

9. Rosa FW, WiLK AL, Kelsey FO. Teratogen update: vitamin A congeners. Teratology 1986; 33: 355-64.

10. KUCERA J. Rate and type of congenital anomalies among offspring of diabetic women. J Reprod Med 1971; 7: 73-82.

11. Emart-Toland A, Yankowitz J, Winder A, Imagire R, Cox VA, Aylsworth AS et al. Oculoauriculoverte- bilateral y una prima paterna, hija de otro tío paterno con una malformación facial no precisada.

En un caso masculino de microtia tipo I, bilateral, su madre y una prima hermana materna tenían la misma característica.

En un caso masculino de Goldenhar con microtia I, su padre y dos tíos paternos presentaban papiloma preauricular.

En un caso masculino de microtia tipo III a derecha, con displasia de caderas a izquierda y Ductus amplio, tiene una abuela paterna con microtia de severidad no precisada.

Dado que la herencia de microtia es autosómica recesiva o autosómica dominante de expresividad variable, al igual que el síndrome de Treacher Collins o el de Goldenhar, podría ser expresiones mínimas, sólo auriculares de ellos.

bral abnormalities in children of diabetic mothers. Am J Med Genet 2000; 90: 303-9.

12. Castima EE, Oriou IM. The Latin American Collaborative Study of Congenital Malformations. Community Genetics 2004; 7: 76-94.

13. ECLAMC Informe Final del ECLAMC 2002 Versión electrónica: http://eclamc.ioc.fiocruz.br/

14. ECLAMC, Manual Operacional. Edición 2002 Ed. Fiocruz. Río de Janeiro. Brasil.

15. International Clearinghouse for Birth Defects Monitoring Systems. Anual report. 1993 Publishes by the ICBD. Roma. Italia.

16. Aase JM, Tegmeier RE. Microtia in New Mexico: Evidence for mulfactorial causation. Birth defects. Original article series, XIII (3 $\left.3^{\text {a }}\right)$ National Foundation Marchof dimes. 1977, pp 113-16.

17. Castila EE, Orioli IM. Prevalence rates of microtia in South America. International Journal of Epidemiology 1986; 15 (3): 364-68.

18. MARTínEz-FriAs ML Defectos congénitos en España: 10 años de vigilancia epidemiológica. Ministerio de Sanidad y Consumo. Madrid España. 1989: 7580.

19. SÁnchez O, Méndez JR, Gómez E, Guerra D. Estudio clínico epidemiológico de la microtia. Invest Clin 1997; 38 (4): 203-17.

20. Shaw GM, Vele EM, Wasserman CR. Risk for neural tube defects affected pregnacies among women of Mexican descent and white women in California. Am J Public Health 1997; 87: 1467-71.

21. Harris JA, Kalien B, Robert E. The epidemiology of anotia and microtia. J Med Genet 1996; 33: 809-13. 